\title{
The Phase-II upgrade of the ATLAS Muon Spectrometer
}

\author{
L. Massa* on behalf of the ATLAS Muon collaboration \\ INFN, Sezione di Roma Tor Vergata - Roma, Italy \\ E-mail: lorenzo.massa@cern.ch
}

The muon spectrometer of the ATLAS detector will undergo a major upgrade foreseen for the Phase II, in order to cope with the operational conditions at the high-luminosity LHC. The trigger and readout electronics for the Resistive Plate Chambers (RPC), Thin Gap Chambers (TGC), and Monitored Drift Tube (MDT) chambers will be replaced to make them compatible with a new trigger scheme with higher trigger rates and longer latencies. MDT precision chambers, that at the moment are not included in the hardware trigger, will be integrated into the level- 0 trigger in order to sharpen the momentum threshold. The MDT front-end electronics will also be replaced. Newgeneration RPC chambers will be installed in the inner barrel layer to increase the acceptance and robustness of the trigger. Some of the MDT chambers in the inner barrel layer will be replaced with new small-diameter MDTs. New TGC triplet chambers in the barrel-endcap transition region will replace the current TGC doublets to suppress the high trigger rate from random coincidences in this region. A major upgrade of the power system is also planned. The Phase-II upgrade concludes the process of adapting the muon spectrometer to the ever increasing performance of the LHC, which started with the Phase-I upgrade New Small Wheel (NSW) project that will replace the innermost endcap wheels.

Sixth Annual Conference on Large Hadron Collider Physics (LHCP2018)

4-9 June 2018

Bologna, Italy

${ }^{*}$ Speaker. 


\section{The Muon Spectrometer and the HL-LHC program}

According to the HL-LHC program foreseen to start in 2026, LHC will reach a maximum luminosity of $L=7.5 \cdot 10^{34} \mathrm{~cm}^{-2} \mathrm{~s}^{-1}$, and a maximum pile-up $\mu=200$, aiming to record between 3000 and $4000 \mathrm{fb}^{-1}$. The conditions of this new phase will allow precision measurements of the Higgs boson properties, in all its main production and decay channels, as well as the study of rare processes and searches for Beyond Standard Model phenomena.

In order to record large statistics of these rare events, triggering muons with a momentum of $20 \mathrm{GeV}$ is fundamental, while the current system would be able to trigger only muons with a momentum greater than $50 \mathrm{GeV}$ at the HL-LHC conditions. Hence, an upgrade of the ATLAS [1] muon spectrometer [2] is needed, allowing a level-0 trigger with a frequency of $1 \mathrm{MHz}$.

Two improvements of the trigger and tracking system of the spectrometer are expected between 2019 and 2020: the inner layer of the End Cap in the $|\eta|>1.3$ region will be replaced by the New Small Wheel [3], while the transition region $(1<|\eta|<1.3)$ of the inner layer of the barrel will be covered by new RPC triplets, the BIS78 project [4].

In addition, further improvements to the detector are foreseen [4].

\subsection{New electronics and trigger system improvement}

The electronics currently mounted in the ATLAS Muon Spectrometer cannot be used for a level-0 trigger able to handle a $1 \mathrm{MHz}$ frequency; hence, a replacement of the DAQ and readout electronics of all the detectors is foreseen. Programmable external FPGAs will be used to make coincidences, instead of the present custom ASICs on detectors, making the new trigger more robust, simple and flexible. Finally, the new trigger will be more redundant and with a sharpened momentum threshold efficiency, adding the information from the precision chambers.

\subsection{Installation of new detectors and power system replacement}

The required performance will be reached by installing new detectors. The acceptance and the efficiency of the trigger will be enlarged by covering the inner barrel (BI) region with triplets of new generation RPCs. To make space for these new triplets, thinner diameter MDTs will replace the present MDTs built on top of the toroid in the inner layer of the muon spectrometer. The present TGC doublets located in the transition region will be replaced by new triplets, in order to reduce the fake trigger rate. Finally, the replacement of the entire power system for low and high voltages is foreseen, as well as the possibility to add a new high- $\eta$ tagger in the $2.7<|\eta|<4.0$ region.

\section{RPC BI Project}

The addition of RPC triplets is one of the most complex projects among the ones foreseen for spectrometer upgrade. During the HL-LHC program, being in an higher luminosity regime, the present ATLAS RPCs will have a working point at a lower voltage, at an efficiency of about $80 \%$, in order not to exceed their safety limits on integrated charge. To recover the efficiency loss and improve selectivity of the trigger, 272 triplets of new generation RPCs will cover the BI region. Furthermore, for the BIS78 project, 32 new triplets will be already installed in 2019 in the transition regions. 
In this way, the entire trigger system will benefit of increased redundancy (9 layers of detectors instead of 6), level-arm (4.5 m instead of $2.3 \mathrm{~m}$ ) and geometrical acceptance ( $96 \%$ instead of $80 \%$ ). The new trigger system will be fully efficient at the luminosities foreseen for HL-LHC, recording events in case of coincidences of 3 chambers out of 4 (instead of 3 out of 3), even with the present RPCs at 70-80\% efficiency, as shown in Figure 1 [4].

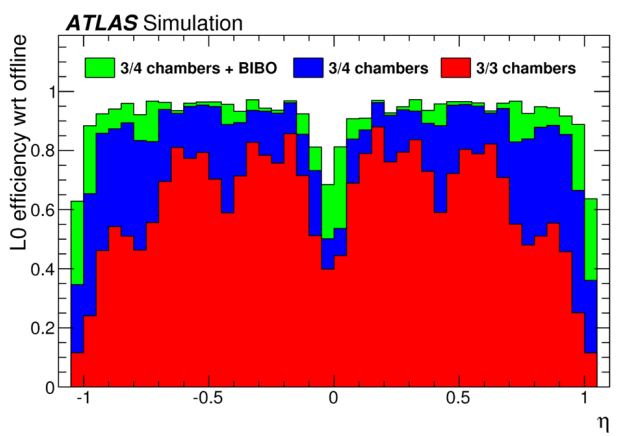

Figure 1: Efficiency times acceptance of the L0 barrel trigger with respect to reconstructed muons with $p_{T}=25 \mathrm{GeV}$ as a function of $\eta$, assuming the worst-case scenario. The histogram shows the efficiency of the existing $3 / 3$ chambers trigger, of the 3/4 chambers trigger including the BI layer, and the additional gain from the BI-BO trigger. Efficiency times acceptance is defined as the fraction of reconstructed muons accepted by the trigger, using a simulation that includes the RPC detector efficiency.

\subsection{New RPCs for Phase-II Upgrade}

\subsubsection{Thinner gas gaps}

The BI triplets will be built using a new generation of RPCs, whose gas gaps and electrodes are both $1 \mathrm{~mm}$ thick, leading to several improvements with respect to the present RPCs mounted in ATLAS having $2 \mathrm{~mm}$ gas gaps and $1.8 \mathrm{~mm}$ electrodes.

Besides important improvements in terms of weight and size, signal collection efficiency and charge distribution, the new thinner dimensions will allow the detector to reach the full efficiency with a lower applied high voltage $(5.4 \mathrm{kV}$ instead of $9.6 \mathrm{kV})$, as shown in Figure 2 (left), and to have an improved time resolution of $0.4 \mathrm{~ns}$ instead of $1 \mathrm{~ns}$ [4].

\subsubsection{Front End electronics in SiGe BiCMOS technology}

The new generation RPCs will use a low-cost, high-performance and low-power front-end board developed in SiGe BiCMOS technology, allowing higher rate capability, more radiation hardness and better space-time resolution with respect to the performance of the present RPCs. The new ASIC will have function of amplifier, discriminator, Time-to-Digital Converter (TDC) and serializer, enhancing the features of the new RPCs with an improved time resolution and the possibility of charge measurements through the time-over-threshold method [4].

The new TDC with serializer, with a time resolution of $100 \mathrm{ps}$, has been prototyped, measuring an intrinsic jitter of $\sigma_{V C O}=\frac{\sigma}{\sqrt{2}}=10.77$ ps [5].

The new preamplifier and discriminator prototypes in SiGe BiCMOS technology have been already produced and tested on $1 \mathrm{~mm}$ RPCs with a high rate muon beam at CERN in October 2017, reaching a time resolution of about $350 \mathrm{ps,} \mathrm{once} \mathrm{the} \mathrm{time-walk} \mathrm{corrections} \mathrm{are} \mathrm{applied,} \mathrm{which} \mathrm{is} \mathrm{the}$ best result ever obtained with these new RPCs [6](Figure 2 (right)). 

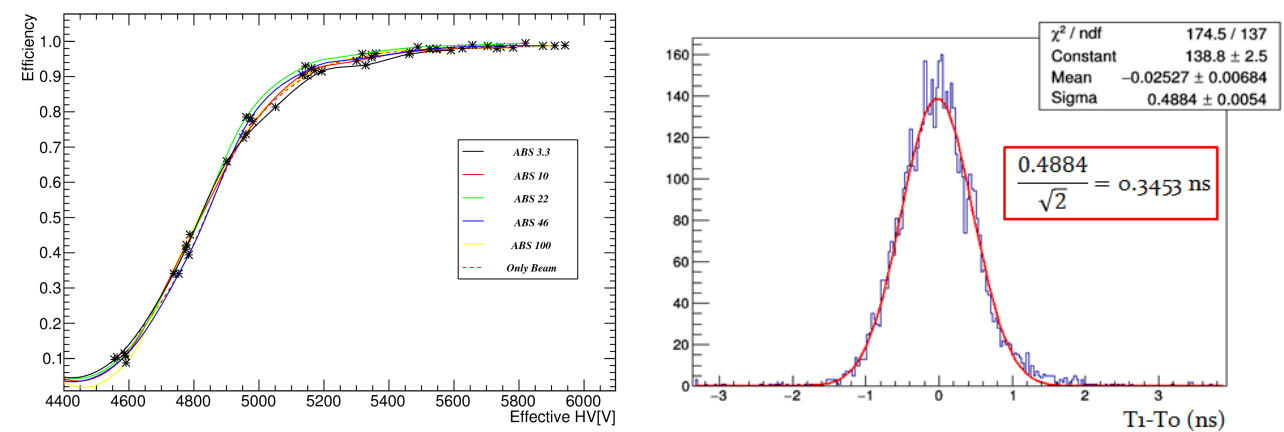

Figure 2: Left: the efficiency plateau for a $1 \mathrm{~mm}$ gap RPC irradiated at the CERN GIF++ with different source intensities. Right: time resolution obtained with a $1 \mathrm{~mm}$ gap RPC and the new discriminator.

\section{Conclusions}

Besides the New Small Wheel and the BIS78 projects foreseen to start data taking in 2021, a further upgrade of the muon spectrometer is planned for the phase 2 upgrade of the ATLAS detector, in order to cope with the operational conditions of the HL- LHC scheduled for 2026.

Among the various detector upgrades which are expected, the BI project will improve the trigger selectivity by adding 272 triplets of new generation of RPCs in the inner layer of the barrel muon spectrometer.

The new detectors which have been developed for this upgrade establish new standards for RPCs in hadron colliders, being already efficient at an applied voltage of $5.4 \mathrm{kV}$, with a time resolution of some hundreds of ps.

Any other additional information can be found in the Technical Design Report [4].

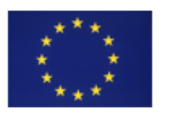

This project has received funding from the European Union's Horizon 2020 Research and Innovation

programme under Grant Agreement no. 654168.

\section{References}

[1] ATLAS Collaboration, The ATLAS Experiment at the CERN Large Hadron Collider, JINST 3 S08003 (2008)

[2] ATLAS Collaboration, ATLAS muon spectrometer: Technical Design Report, CERN-LHCC-97-022, https://cds.cern.ch/record/331068 (1997)

[3] ATLAS Collaboration, New Small Wheel Technical Design Report, CERN-LHCC-2013-006, https://cds.cern.ch/record/1552862 (2013)

[4] ATLAS Collaboration, Technical Design Report for the Phase-II Upgrade of the ATLAS Muon Spectrometer, CERN-LHCC-2017-017, https://cds.cern.ch/record/2285580 (2017)

[5] S.Bruno et al., Design a TDC in SiGe for the Front-end electronics for the RPCs used in a high-rate experiment, arXiv:1806.04082 [physics.ins-det] (2018)

[6] L.Pizzimento et al., Development of a new Front-End electronics in Si and Si-Ge technology for the Resistive Plate Chamber (RPC) detector for high rate experiments, arXiv:1806.04113

[physics.ins-det] (2018) 